\title{
Structural and Magnetic Characterization of B20 Skyrmion Thin Films and Heterostructures Using Aberration-Corrected Lorentz TEM and Differential Phase Contrast STEM
}

\author{
B. D. Esser, ${ }^{1}$ A. S. Ahmed, ${ }^{2}$ R. K. Kawakami, ${ }^{2}$ and D. W. McComb ${ }^{1}$
}

1. Center for Electron Microscopy and Analysis, The Ohio State University, Columbus, OH, USA

2. Department of Physics, The Ohio State University, Columbus, OH, USA

Magnetic materials exhibiting topological spin textures have shown great promise for magnetoelectronic applications including ultra-high density magnetic memory. [1-4] Specifically, skyrmions are vortex-like spin textures that can form hexagonal magnetic lattices at temperatures near room temperature and small applied magnetic fields. The skyrmion phase results from the competition between exchange interactions and the Dzyaloshinskii-Moriya (DM) interaction, where the exchange interaction promotes parallel alignment between neighboring spins and the DM interaction promotes $90^{\circ}$ alignments. DM interactions only occur in structures with broken inversion or mirror symmetry like the family of materials with the B20 crystal structure (space group P2 3 ). In addition to materials lacking in bulk inversion or mirror symmetry, superlattices can host the skyrmion phase due to their broken mirror symmetry. Recently, Ahmed et al. demonstrated the ability grow epitaxial B20 superlattices of $[\mathrm{CrGe} / \mathrm{MnGe} / \mathrm{FeGe}]_{n}$ via molecular beam epitaxy (MBE) opening the door for tunable skyrmions through varying layer thicknesses. [5]

To fully understand the magnetic properties of these materials, atomic resolution imaging, energy dispersive X-ray spectroscopy (EDX), and in situ Lorentz transmission electron microscopy (LTEM) and differential phase contrast scanning transmission electron microscopy (DPC-STEM) were performed on FeGe bulk crystals and heterostructures of $[\mathrm{FeGe} / \mathrm{CrGe}]_{n}$, and $[\mathrm{CrGe} / \mathrm{MnGe} / \mathrm{FeGe}]_{n}$. Atomic resolution imaging performed on a probe corrected FEI Titan ${ }^{3}$ 80-300 S/TEM revealed clear B20 structure and EDX demonstrates the quality of the interfaces with very little interdiffusion between layers in the heterostructures. LTEM and DPC-STEM were performed on an image corrected FEI Titan ${ }^{3}$ G2 60-300 $\mathrm{S} / \mathrm{TEM}$ in variable temperature and applied field. For both the LTEM and DPC-STEM imaging modes, the objective lens field was lowered to nearly $0 \mathrm{~T}$ in the plane of the specimen and then reapplied in situ up to approximately $150 \mathrm{mT}$ and at temperatures ranging from $90 \mathrm{~K}$ to above the magnetic ordering temperature of $280 \mathrm{~K}$. In LTEM, the resulting magnetic structure results in image contrast due to the inplane Lorentz force which inverts as a function of defocus. In DPC-STEM, the in-focus probe is rastered across the specimen and images are formed using a segmented quadrant annular detector in the diffraction plane. This allows for the detection of small shifts in the diffraction pattern due to the inplane Lorentz force yielding similar results to LTEM but with higher spatial resolution. Both techniques were used to quantitatively study the in-plane magnetization in the skyrmion systems to fully understand the roles of temperature, field, specimen thickness, and microstructure in the resultant magnetic structure.

In this work, we start with characterization of the well-studied case of FeGe bulk crystals, and then progress through the more novel heterostructures of $[\mathrm{FeGe} / \mathrm{CrGe}]_{n}$ and $[\mathrm{CrGe} / \mathrm{MnGe} / \mathrm{FeGe}]_{n}$ demonstrating the potential to tune skyrmion size through precise materials engineering. A suite of stateof-the-art microscopy-based characterization techniques are employed to better understand and advance this class of materials with major potential applications in magnetic storage and computation. 


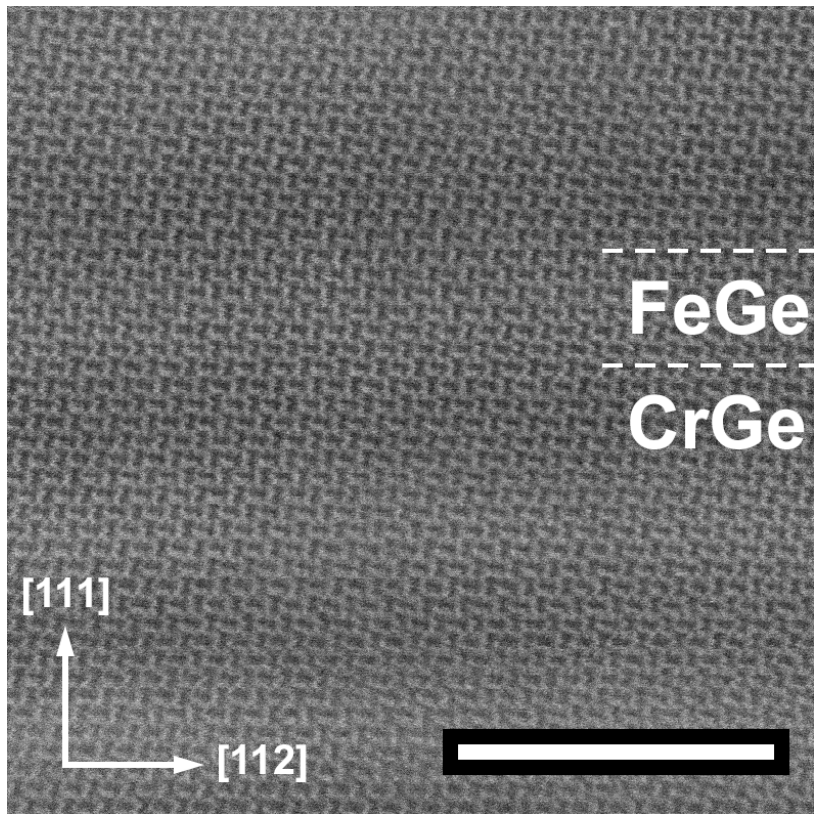

Figure 1: High angle annular dark field STEM image of [11̄0] [FeGe/CrGe] heterostructure demonstrating clear B20 structure, coherent interfaces between the two different layers, and minimal interdiffusion. Scale bar is $5 \mathrm{~nm}$.
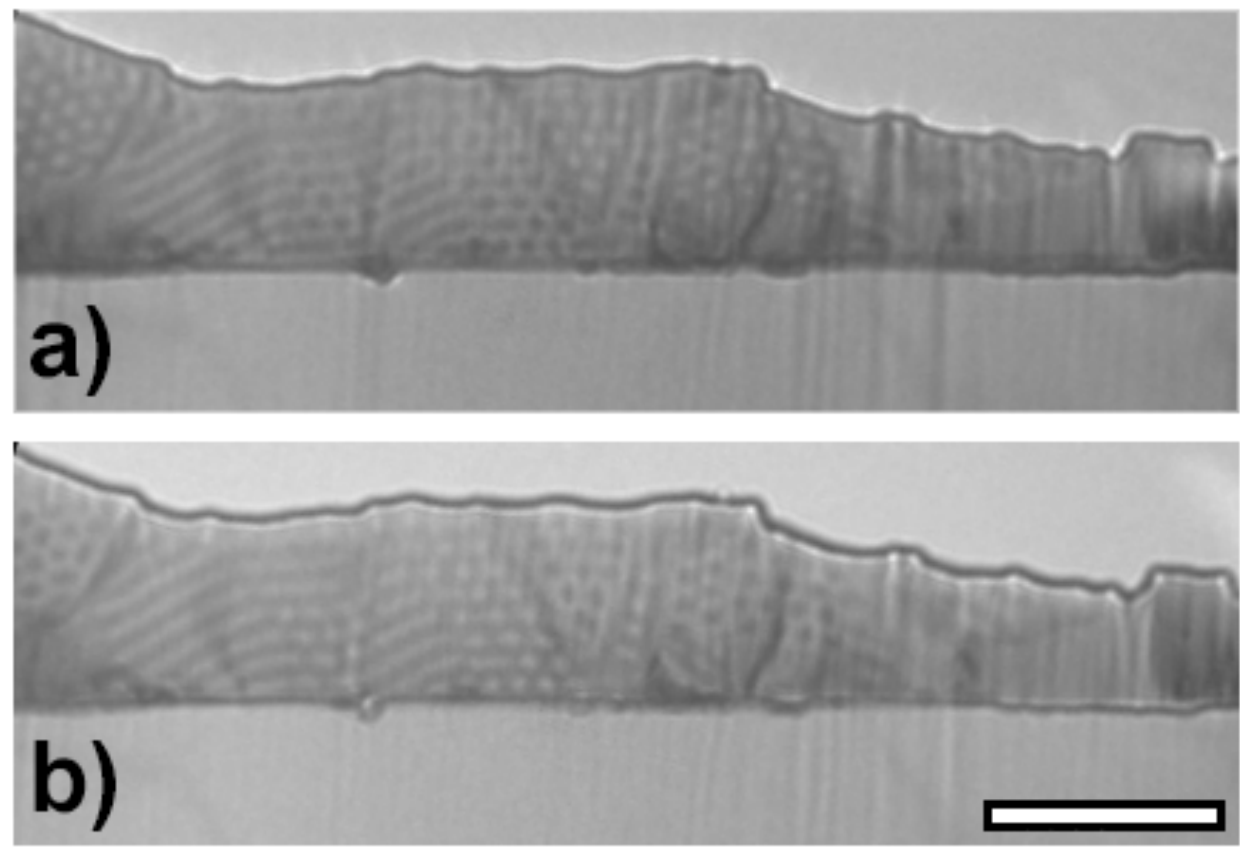

Figure 2: Aberration corrected LTEM image of [11̄0] FeGe thin film on (111)Si substrate at 256K and $40 \mathrm{mT}$ applied field. Skyrmion lattice is visible in a) $+178 \mu \mathrm{m}$ and b) $-178 \mu \mathrm{m}$ defocus highlighting the reversal of magnetic contrast with defocus. Scale bar is $1 \mu \mathrm{m}$.

[1] M. C. Langner et al., Physical Review Letters 112, 167202 (2014).

[2] T. Schulz et al., Nature Physics 8, 301 (2012).

[3] P. Milde et al., Science 340, 1076 (2013).

[4] S. Woo et al., Nature Materials 15, 501-506 (2016).

[5] A. S. Ahmed et al., arXiv:1702.05191 (2017).

[6] Funding for this research was provided by the Center for Emergent Materials at the Ohio State University, an NSF MRSEC (Award Number DMR-1420451), as well as from the Ohio State Materials Seed Grants (MTB-G00010 and MTB-G00012). 